\title{
The role of vitamin $D$ in the aetiology of type 1 diabetes
}

\author{
Olle Korsgren ${ }^{1,2}$ (i)
}

Received: 20 January 2020 / Accepted: 22 January 2020 / Published online: 10 February 2020

(C) Springer-Verlag GmbH Germany, part of Springer Nature 2020

Abbreviations

25OHD 25-Hydroxyvitamin D

To the Editor: The role of vitamin D in the development of type 1 diabetes remains unresolved over several decades. In the communication by Miettinen et al. [1] further evidence is provided arguing for a critical role of early postnatal vitamin D for protection against the development of type 1 diabetes, as evidenced by a small but significantly decreased level of serum 25-hydroxyvitamin D (25OHD) concentration in blood samples of case children (defined as having positivity for at least two out of four diabetes-associated autoantibodies) compared with those of country- and age-matched controls ( 58.0 vs $65.0 \mathrm{nmol} / 1, p=0.018$, measured 18 months prior to the age of first seroconversion). Notably, only $3 \%$ of the case children and $2 \%$ of the control children had severe vitamin D deficiency at any time point (serum 25OHD concentration $<30 \mathrm{nmol} / \mathrm{l})$.

Vitamin D is lipophilic and it is mainly absorbed in the small intestine before further processing in the skin, liver and kidneys to the biological active compound 1,25 dihydroxyvitamin D. Absorption of lipophilic substances is dependent on a variety of intricate processes requiring an intact epithelium in the small intestine, but also on extraintestinal factors, such as the release of lipase from the pancreas and bile from the liver [2].

Type 1 diabetes is an inflammatory pancreatic disease that causes, already in the early disease process, a slight malabsorption, particularly of lipophilic substances [3]. The underlying mechanism for this is at present unknown but can probably be attributed to smaller than expected pancreatic

Olle Korsgren

olle.korsgren@igp.uu.se

1 Department of Immunology, Genetics and Pathology, Rudbeck Laboratory, Uppsala University, 75185 Uppsala, Sweden

2 Department of Clinical Chemistry and Transfusion Medicine, Institute of Biomedicine, University of Gothenburg, Gothenburg, Sweden parenchyma as well as signs of subclinical pancreatitis and ongoing inflammation dominated by monocytes and neutrophils [3]. Notably, the pancreatic volume is also reduced in first degree relatives of individuals with type 1 diabetes when compared with control participants with no family history of type 1 diabetes [4].

A concomitant morbidity of type 1 diabetes is coeliac disease. In Sweden about $5 \%$ of children newly diagnosed with type 1 diabetes already have increased titres of tissue transglutaminase antibodies at the time of diagnosis of diabetes [5]. The presence of these autoantibodies provides high positive predictive value of coeliac disease. International systematic reviews show that $3-5 \%$ of individuals diagnosed with type 1 diabetes have biopsy-confirmed coeliac disease (see for example [6]). Type 1 diabetes seems to precede the development of coeliac disease by a few years, and most participants developing coeliac disease are diagnosed within the first 2 years after diabetes diagnosis [6]. Coeliac disease adversely affects the epithelium of the small intestine and causes an impairment in the absorption of vitamin D, among other things. Even in affected people eating an appropriate diet for coeliac disease vitamin D deficiency can often be detected, showing the importance of the diet and a physiologically intact intestinal epithelium in the absorption of the lipophilic substance [7].

The effect of vitamin D deficiency on the cells of the immune system and the expression of the vitamin D receptor on beta cells is often taken to support a mechanistic involvement of vitamin $\mathrm{D}$ in the development of type 1 diabetes. However, epidemiological data seem to be in conflict with this notion; vitamin $\mathrm{D}$ deficiency is particularly common in countries with low incidence of type 1 diabetes, e.g. China and Iran. Furthermore, in Europe, vitamin D deficiency has been described as pandemic with $13.0 \%$ of the 55,844 examined European individuals having serum 25OHD concentrations below $30 \mathrm{nmol} / \mathrm{l}$, but with lower frequency of vitamin D deficiency in Nordic countries, i.e. the countries with the highest frequency of type 1 diabetes [8]. Notably, individuals diagnosed with classical vitamin D deficiency, other than from pancreatic causes, do not usually develop type 1 diabetes even if untreated for such prolonged periods as to cause rickets [2]. 
As elegantly shown in the communication by Miettinen et al. [1] there is an association in population studies between a slight decrease in the level of circulating vitamin D and autoantibody positivity early in disease progression to type 1 diabetes. However, association is not evidence of causality and other known comorbidities of type 1 diabetes, such as those discussed above, should be considered to provide as complete a view as possible on the role of vitamin D in type 1 diabetes.

Authors' relationships and activities The author declares that there are no relationships or activities that might bias, or be perceived to bias, their work.

Contribution statement The author was the sole contributor to this paper.

\section{References}

1. Miettinen ME, Niinistö S, Erlund I et al (2020) Serum 25hydroxyvitamin D concentration in childhood and risk of islet autoimmunity and type 1 diabetes: the TRIGR nested case-control ancillary study. Diabetologia. https://doi.org/10.1007/s00125-01905077-4

2. Holick MF (2007) Vitamin D deficiency. N Engl J Med 357:266-281

3. Alexandre-Heymann L, Mallone R, Boitard C, Scharfmann R, Larger E (2019) Structure and function of the exocrine pancreas in patients with type 1 diabetes. Rev Endocr Metab Disord 20:129-149

4. Campbell-Thompson ML, Filipp SL, Grajo JR et al (2019) Relative pancreas volume is reduced in first-degree relatives of patients with type 1 diabetes. Diabetes Care 42:281-287

5. Cerqueiro Bybrant M, Grahnquist L, Ortqvist E et al (2018) Tissue transglutaminase autoantibodies in children with newly diagnosed type 1 diabetes are related to human leukocyte antigen but not to islet autoantibodies: a Swedish nationwide prospective populationbased cohort study. Autoimmunity 51:221-227

6. Craig ME, Prinz N, Boyle CT et al (2017) Prevalence of celiac disease in 52,721 youth with type 1 diabetes: international comparison across three continents. Diabetes Care 40:1034-1040

7. Di Nardo G, Villa MP, Conti L et al (2019) Nutritional deficiencies in children with celiac disease resulting from a gluten-free diet: a systematic review. Nutrients:11

8. Cashman KD, Dowling KG, Skrabakova Z et al (2016) Vitamin D deficiency in Europe: pandemic? Am J Clin Nutr 103:1033-1044

Publisher's note Springer Nature remains neutral with regard to jurisdictional claims in published maps and institutional affiliations. 